\title{
On the structure of globular cluster systems in elliptical galaxies
}

\author{
K. Bekki ${ }^{1}$ and D. A. Forbes ${ }^{2}$ \\ 1 School of Physics, University of New South Wales, Sydney 2052, NSW, Australia \\ e-mail: bekki@bat.phys.unsw.edu.au \\ 2 Centre for Astrophysics and Supercomputing, Swinburne University of Technology, Mail H39, PO Box 218, Hawthorn, VIC 3122, Australia \\ e-mail: dforbes@astro.swin.edu.au
}

Received 23 June 2005 / Accepted 14 September 2005

\section{ABSTRACT}

It has long been known that the radial density profiles of globular cluster systems (GCSs) in elliptical galaxies vary with the total luminosities of their host galaxies. In order to elucidate the origin of this structural non-homology in GCSs, we numerically investigate the structural properties of GCSs in elliptical galaxies formed from a sequence of major dissipationless galaxy merging. We find that the radial density profiles of GCSs in elliptical galaxies become progressively flatter as the galaxies experience more major merger events. The density profiles of GCSs in ellipticals are well described as power-laws with slopes $\left(\alpha_{\mathrm{gc}}\right)$ ranging from -2.0 to -1.0 . They are flatter than, and linearly proportional to, the slopes $\left(\alpha_{\mathrm{s}}\right)$ of the stellar density profiles of their host galaxies. We also find that the GCS core radii $\left(r_{\mathrm{c}}\right)$ of the density profiles are larger in ellipticals that experienced more mergers. By applying a reasonable scaling relation between luminosities and sizes of galaxies to the simulation results, we show that $\alpha_{\mathrm{gc}} \approx-0.36 M_{\mathrm{V}}-9.2, r_{\mathrm{c}} \approx-1.85 M_{\mathrm{V}}$, and $\alpha_{\mathrm{gc}} \approx 0.93 \alpha_{\mathrm{s}}$, where $M_{\mathrm{V}}$ is the total $V$-band absolute magnitude of a galaxy. We compare these predictions with observations and discuss their physical meaning. We suggest that the origin of structural non-homology of GCSs in ellipticals can be understood in terms of the growth of ellipticals via major dissipationless galaxy merging.

Key words. Galaxy: globular clusters: general - galaxies: star clusters - galaxies: elliptical and lenticular, cD - galaxies: evolution galaxies: interactions

\section{Introduction}

The structural properties of globular cluster systems (GCSs) in galaxies have long been suggested to contain fossil information on the early dynamical histories of galaxies and has accordingly been investigated both observationally and theoretically (e.g., Harris 1986, 1991). In particular, radial density profiles of GCSs and their correlations with physical properties of their host galaxies have been discussed in terms of formation and evolution of elliptical galaxies (e.g., Harris 1986; Zepf \& Ashman 1993; Ashman \& Zepf 1998; Forbes et al. 1996, 1997; Bekki et al. 2002, 2003). In particular, observational studies found that the GCSs in early-type galaxies were less centrally concentrated than the stellar light of the host galaxy (e.g., Harris \& Racine 1979; Strom et al. 1981; Grillmair et al. 1994; Forbes et al. 1996), and that the outer slope of the GCS density profile correlated with the host galaxy luminosity (e.g., Ashman \& Zepf 1998; Harris 1986).

Major galaxy merging between equal-mass disk galaxies has long been considered to be a promising formation scenario for elliptical galaxy formation (e.g., Toomre 1977). Although this galaxy formation scenario has been discussed in many contexts (e.g., the color-magnitude relation), it has not been extensively investigated in terms of whether it can self-consistently explain the observed properties of GCSs around ellipticals. So far, only the observed higher specific frequency $\left(S_{\mathrm{N}}\right)$ and bimodal color distributions of GCSs have been discussed in terms of hierarchical galaxy formation (Beasley et al. 2002). It is thus unknown what implications the observed structural properties of GCSs in ellipticals has for galaxy formation.

The purpose of this paper is to propose that the radial density profiles of GCSs in elliptical galaxies have fossil information on how often ellipticals have experienced major merger events in their dynamical histories. By using dissipationless N-body simulations of major merging of galaxies, we investigate how the structural properties of GCSs in elliptical galaxies change as they grow by successive (gas-free) mergers. We compare our theoretical predictions with observations to discuss the origin of the observational trends. We also briefly discuss the role of GC accretion. In particular, we focus on the luminosity-dependence of the GCS slope $\left(\alpha_{\mathrm{gc}}\right)$ which implies a "non-homology" in the structural properties of GCSs.

\section{Model}

We investigate the dynamical evolution of GCSs of elliptical galaxies formed from sequential dissipationless major merging based on numerical simulations carried out on the GRAPE board (Sugimoto et al. 1990) in GRAPE 5 systems. We here 
stress that the roles of minor merging/accretion of GC in shaping radial density profiles of GCSs in galaxies are not extensively investigated in the present models. We adopt the following merging scheme to model elliptical galaxy formation through sequential major equal-mass disk mergers. First an elliptical galaxy is formed by major merging between two equalmass disk galaxies. We refer to the remnant elliptical galaxy as the 1st-generation elliptical. This 1st-generation elliptical then merges with other 1st-generation one to form the 2ndgeneration elliptical. This sequential major merging can repeat for $N_{\max }$ times, and the structural properties of stellar components and GCSs are investigated for each $i$ th generation elliptical ( $i \leq N_{\max }$ ). The total particle number used is 27670 for the simulations of the 1st-generation elliptical and 221360 for those of the 4 th-generation one (i.e., $N_{\max }$ of 4 ). The initial total particle number for GCs is set to be 1000 , because an order of 1000 (not 10 or 100) is necessary to make robust predictions for the radial profiles of structure and kinematics of GCSs in galaxies (Bekki et al. 2005).

Since the numerical methods and techniques we employ for modeling dynamical evolution of galaxy mergers with GCs have already been described in detail elsewhere (Bekki et al. 2002, 2005), we give only a brief review here. The progenitor disk galaxies that take part in a merger (for the 1st-generation elliptical) are assumed to have a dark halo, a bulge, a stellar halo, a thin exponential disk, and a GCS. The total disk mass and size are $M_{\mathrm{d}}$ and $R_{\mathrm{d}}$, respectively. Henceforth, all masses are measured in units of $M_{\mathrm{d}}$ and distances in units of $R_{\mathrm{d}}$, unless otherwise specified. We adopt the density distribution of the NFW halo (Navarro et al. 1996) suggested from CDM simulations:

$\rho(r)=\frac{\rho_{0}}{\left(r / r_{\mathrm{s}}\right)\left(1+r / r_{\mathrm{s}}\right)^{2}}$,

where $r, \rho_{0}$, and $r_{\mathrm{s}}$ are the spherical radius, the central density of a dark halo, and the scale length of the halo, respectively. The dark matter to disk mass ratio is fixed at 9 for all models. The value of $r_{\mathrm{S}}$ (typically $\sim 3 R_{\mathrm{d}}$ ) is chosen such that the rotation curve of the disk is reasonably consistent with observations for a given bulge mass. We adopt the $R^{1 / 4}$ profile for the bulge with the mass of 0.17 and a scale length of $0.04 R_{\mathrm{d}}$. The radial $(R)$ and vertical $(Z)$ density profiles of the disk are assumed to be proportional to $\exp \left(-R / R_{0}\right)$ with scale length $R_{0}=0.2 R_{\mathrm{d}}$ and to $\operatorname{sech}^{2}\left(Z / Z_{0}\right)$ with scale length $Z_{0}=0.04 R_{\mathrm{d}}$ in our units, respectively.

The GCSs in the progenitor spirals have a spherical distribution with a density profile of $\rho(r) \propto r^{\alpha_{\mathrm{gc}, \mathrm{i}}}$. We adopt $\alpha_{\mathrm{gc}, \mathrm{i}}=-3.5$ for most models (however the dependence of the final radial profiles of GCSs in mergers on $\alpha_{\mathrm{gc}, \mathrm{i}}$ is also investigated). The adopted density profile of $\rho(r) \propto r^{-3.5}$ is consistent with that observed for the Galactic GCS (Djorgovski \& Meylan 1994). The GCS of M 31 reveals a similar surface density distribution to the Milky Way GCS (e.g. Battistini et al. 1993). Very little is known about the density profile of GCSs in other spirals. The velocity dispersion of a GCS is assumed to be isotropic which is consistent with the observations of the Galactic GCS (e.g., Freeman 1993).

The simulations are dissipationless so no new GCs form in the merging process. Thus our simulations are more appropriate for relatively gas-free mergers which may be expected to occur at some late epochs. Recently, observational studies by Bell et al. (2005) have demonstrated that present day spheroidal galaxies with $M_{\mathrm{V}}<-20.5$ on average have undergone between 0.5 and 1 major "dry merger" (i.e., gas-poor mergers) since $z \sim 0.7$. This observational paper thus suggests that the dissipationless merger models adopted in the present paper can be reasonable for elliptical galaxy formation. ProtoGCs have been observed to form in late epoch mergers (e.g. Whitmore \& Schweizer 1995), which implies that some fraction of GCs in ellipticals could be quite young. However, latest observational studies (e.g. Cohen et al. 1998, 2003; Forbes et al. 2001; Kuntschner et al. 2002; Beasley et al. 2004; Strader et al. 2004; Pierce et al. 2005), in which more reliable age estimation of GCs can be done based on the higher resolution spectra and the improved stellar population synthesis model, have demonstrated that the vast bulk of GCs in early-type galaxies are very old, i.e. $\geq 10$ Gyrs. These suggest that pre-existing GCs dominate in old merger remnants.

In all of the simulations, the orbit of the two galaxies is set to be initially in the $x y$ plane and the distance between the center of mass of the two is assumed to be 6 in our units. The pericenter distance $\left(r_{\mathrm{p}}\right)$ and the eccentricity $\left(e_{\mathrm{p}}\right)$ in a merger are assumed to be free parameters that control orbital energy and angular momentum of the merger. The spin of each galaxy in a merger is specified by two angles $\theta_{i}$ and $\phi_{i}$, where suffix $i$ is used to identify each galaxy. Here $\theta_{i}$ is the angle between the $z$ axis and the vector of the angular momentum of a galaxy, and $\phi_{i}$ is the azimuthal angle measured from the $x$ axis to the projection of the angular momentum vector of a galaxy onto the $x y$ plane. We specifically show four different and representative models of merger sequences with $N_{\max }=4$ in which merger pairs take one of the following orbital configurations (i.e., galaxy inclinations with respect to the orbital plane): A prograde-prograde model represented by "PP" with $\theta_{1}=0$, $\theta_{2}=30, \phi_{1}=0, \phi_{2}=0, r_{\mathrm{p}}=1.0$, and $e_{\mathrm{p}}=0.72$ a retrograderetrograde ("RR") with $\theta_{1}=180, \theta_{2}=150, \phi_{1}=0, \phi_{2}=0$, $r_{\mathrm{p}}=1.0$, and $e_{\mathrm{p}}=0.72$ and a highly inclined model ("HI") with $\theta_{1}=30, \theta_{2}=120, \phi_{1}=90$, and $\phi_{2}=180, r_{\mathrm{p}}=1.0$, $e_{\mathrm{p}}=1.0$ and the low orbital angular momentum model ("LA") with $\theta_{1}=30, \theta_{2}=120, \phi_{1}=90$, and $\phi_{2}=180, r_{\mathrm{p}}=0.2$, $e_{\mathrm{p}}=1.0$.

In analyzing the projected radial density profiles of GCs $\left(\Sigma_{\mathrm{gc}}\right)$ and stars $\left(\Sigma_{\mathrm{s}}\right)$, we assume that they are approximated as $\Sigma_{\mathrm{gc}} \propto R^{\alpha_{\mathrm{gc}}}$ and $\Sigma_{\mathrm{s}} \propto R^{\alpha_{\mathrm{s}}}$, where $R$ is the projected distance from the center of a galaxy. We derive the slopes of $\alpha_{\mathrm{gc}}$ and $\alpha_{\mathrm{s}}$ and the difference of the two $\alpha_{\mathrm{gc}}-\alpha_{\mathrm{s}}$, for $5 \leq R(\mathrm{kpc}) \leq 20$ so that the derived values can be directly compared with observations. We determine a core radius $\left(r_{\mathrm{c}}\right)$ for the GCS by adopting an isothermal profile of the form $\Sigma_{\mathrm{gc}} \propto\left(r^{2}+{r_{\mathrm{c}}}^{2}\right)^{-1}$, which can be directly compared to the observations of Forbes et al. (1996). For an isothermal profile $r_{\mathrm{c}} \approx 0.22 R_{\mathrm{h}, \mathrm{gc}}$, where $R_{\mathrm{h}, \mathrm{gc}}$ is a halfnumber radius of the GCS. In order to estimate the total $V$-band magnitude of a merger remnant, we assume an initial disk mass of $M_{\mathrm{d}}=4.0 \times 10^{10} M_{\odot}$, an initial disk size of $R_{\mathrm{d}}=14 \mathrm{kpc}$ and $M_{\mathrm{d}} / L_{\mathrm{V}}=5.0$, where $L_{\mathrm{V}}$ is the total $V$-band luminosity of the initial disk. The remnant's total mass is $2^{N_{\mathrm{m}}} \times M_{\mathrm{d}}$, where $N_{\mathrm{m}}$ is 




Fig. 1. Dependences of projected number distributions of stars (thin) and GCs (thick) in merger remnants (i.e., elliptical galaxies) on the total number of major merger events $\left(N_{\mathrm{m}}\right)$ which an elliptical experienced during its formation. For clarity, the density distributions are normalized to their central values. Thin dotted lines represent powerlaw slopes $(\alpha)$ of $\alpha=-2.5,-2.0,-1.5$, and -1.0 . Note that the density profiles of GCSs become flatter for larger $N_{\mathrm{m}}$, i.e. more mergers.

total number of major merger events that the remnant elliptical experienced.

All the calculations related to the above dissipationless evolution have been carried out on a GRAPE board (Sugimoto et al. 1990) in the GRAPE 5 system at the National Astronomical Observatory of Japan. The parameter of gravitational softening for stellar particles is set to be fixed at 0.039 in our units $(0.68 \mathrm{kpc})$. The time integration of the equation of motion is performed by using the 2nd-order leap-flog method.

\section{Results}

\subsection{Structural non-homology}

Figure 1 plots the radial density profiles of stars and GCs in ellipticals variation with $N_{\mathrm{m}}$ in the $\mathrm{HI}$ model sequence. The reference GC profile with $\alpha_{\mathrm{gc}}=-2.5$ in this figure corresponds to the Galactic GC profile (Zinn 1985) which shows $\alpha_{\mathrm{gc}} \sim-2$ out to $R=10 \mathrm{kpc}$ and $\alpha_{\mathrm{gc}} \sim-3$ beyond $R=20 \mathrm{kpc}$ (Harris 1976). This figure shows that up to $N_{\mathrm{m}}=4$, the ellipticals have positive $\alpha_{\mathrm{gc}}-\alpha_{\mathrm{s}}$ (e.g. 0.58 for $N_{\mathrm{m}}=1$ ), and thus show flatter profiles for GCs than for field stars, which is consistent qualitatively with observations (e.g., Harris 1986, 1991). The reason

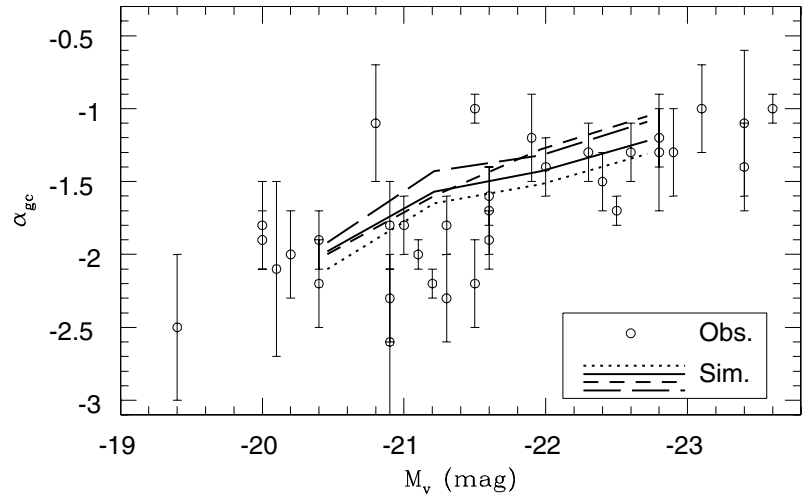

Fig. 2. Comparison between the observed $\alpha_{\mathrm{gc}}-M_{\mathrm{V}}$ relation and the simulated one. The observational data, represented by open circles, come from the table in the appendix of Ashman \& Zepf (1998). Solid, dotted, short-dashed, and long-dashed lines show the results of the HI, PP, RR, and LA models, respectively. Note that both simulations and observations show a tendency for flatter density profiles in brighter ellipticals.

for this is that GCs in a merger are more "puffed up" relative to the stars after they inevitably absorb orbital angular momentum and kinetic energy during the merger, because most GCs are initially located in the galactic outer parts where such absorption is most likely to occur. The radial density profile of a GCS in an elliptical is always flatter in the inner region $(R<20 \mathrm{kpc})$ than in the outer one $(R \geq 20 \mathrm{kpc})$. Again, qualitatively consistent with observations (e.g., Forbes et al. 1996).

Both stellar and GC profiles in an elliptical become progressively flatter as the elliptical experiences more major merger events (i.e., larger $N_{\mathrm{m}}$ ). For example, in the HI model $\alpha_{\mathrm{gc}}$ is estimated as -1.99 for $N_{\mathrm{m}}=1$ and -1.23 for $N_{\mathrm{m}}=4$, which implies that the slope $\alpha_{\mathrm{gc}}$ of a GCS in an elliptical has fossil information on how many times the elliptical has experienced dissipationless major merger events in its formation history. The derived slopes for ellipticals are generally flatter than that observed for the Galactic GCS which has $\alpha_{\mathrm{gc}} \approx-2.5$ (Zinn 1985). This suggests that major merging can transform the initially steeper density profiles of GCSs in spirals into the flatter ones observed in ellipticals. The general trends shown in Fig. 1 do not depend on model parameters.

Figure 2 shows how $\alpha_{\mathrm{gc}}$ depends on the total $V$-band magnitude of ellipticals for four different sets of models (i.e., PP, RR, HI, and LA). Irrespective of the models, GCSs in brighter ellipticals show flatter density profiles (i.e., larger $\alpha_{\mathrm{gc}}$ ), which is reasonably consistent with observations. This is due to the fact that $\alpha_{\mathrm{gc}}$ becomes larger each time a major merger occurs. A least square fit to the simulation data shown in Fig. 2 gives $\alpha_{\mathrm{gc}} \approx-0.36 M_{\mathrm{V}}-9.2$, which is similar to the observed relation of $\alpha_{\mathrm{gc}} \propto-0.3 M_{\mathrm{V}}$ (Harris 1986). This result suggests that the origin of the luminosity-dependent $\alpha_{\mathrm{gc}}$ (Harris 1991; Ashman $\&$ Zepf 1998) can be understood in terms of the growth of ellipticals via major dissipationless mergers. It should be however noted, that the simulated range of $\alpha_{\mathrm{gc}}$, for a given luminosity, is quite narrow so that the present models can not simply explain the scatter observed for $\alpha_{\mathrm{gc}}$ (in particular, for $\left.-22<M_{\mathrm{V}}<-21\right)$. 


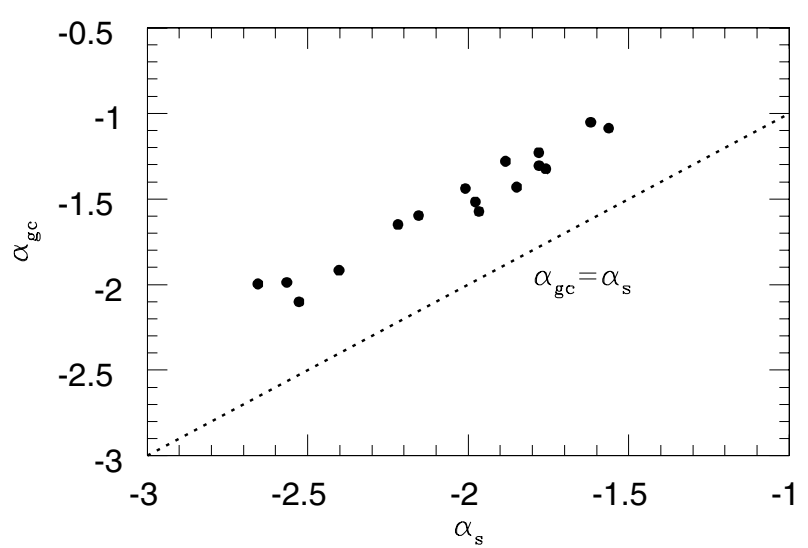

Fig. 3. The dependence of the GCS profile slope $\left(\alpha_{\mathrm{gc}}\right)$ and that for the galaxy stars $\left(\alpha_{\mathrm{s}}\right)$ in 16 simulated merger remnants, i.e. elliptical galaxies. The dotted line indicates $\alpha_{\mathrm{gc}}=\alpha_{\mathrm{s}}$.



Fig. 4. Comparison between the observed $r_{\mathrm{c}}-M_{\mathrm{V}}$ and the simulated ones. Here $r_{\mathrm{c}}$ is the core radius of a GCS's distribution. The observational data represented by open circles come from Forbes et al. (1996). Solid, dotted, short-dashed, and long-dashed line show the results of the HI, PP, RR, and LA models, respectively.

Figure 3 shows a positive correlation between $\alpha_{\mathrm{gc}}$ and $\alpha_{\mathrm{s}}$ $\left(\alpha_{\mathrm{gc}} \approx 0.93 \alpha_{\mathrm{s}}+0.37\right)$, which means that ellipticals with flatter distributions of stars are highly likely to show flatter distributions of GCSs. Figure 3 also shows that $\alpha_{\mathrm{s}}$ is always smaller than $\alpha_{\mathrm{gc}}$ (i.e., $\alpha_{\mathrm{gc}}-\alpha_{\mathrm{s}} \sim 0.4$ ) and thus confirms that elliptical galaxies formed by major merging can have GCSs with the density profiles flatter than those of stars. The density profiles of the GCSs can be fit by a power-law with slope $\alpha_{\mathrm{gc}} \approx-0.4$ for the inner region of $0.5 \leq R \leq 10 \mathrm{kpc}$. However the shallower profiles of GCSs in the inner regions can not be regarded as "flat cores" $\left(\alpha_{\mathrm{gc}} \sim 0\right)$ which appear to be common in the GCSs of giant ellipticals (e.g., Harris 1986, 1991; Forbes et al. 1996).

Figure 4 shows a clear trend of larger GCS core radii $\left(r_{\mathrm{c}}\right)$ for brighter ellipticals, which is consistent qualitatively with the observational results of Forbes et al. (1996) for the overall GCS. The derived trend is due to the fact that $r_{\mathrm{c}}$ becomes larger by a factor of $\sim 1.4$ after each major merger. We can estimate the luminosity-dependence of $r_{\mathrm{c}}$ as $r_{\mathrm{c}} \propto-1.41 M_{\mathrm{V}}$ for the observational data with $M_{\mathrm{V}} \leq-20.5$ of Forbes et al. (1996), and as $r_{\mathrm{c}} \propto-1.85 M_{\mathrm{V}}$ for the simulation data set shown in

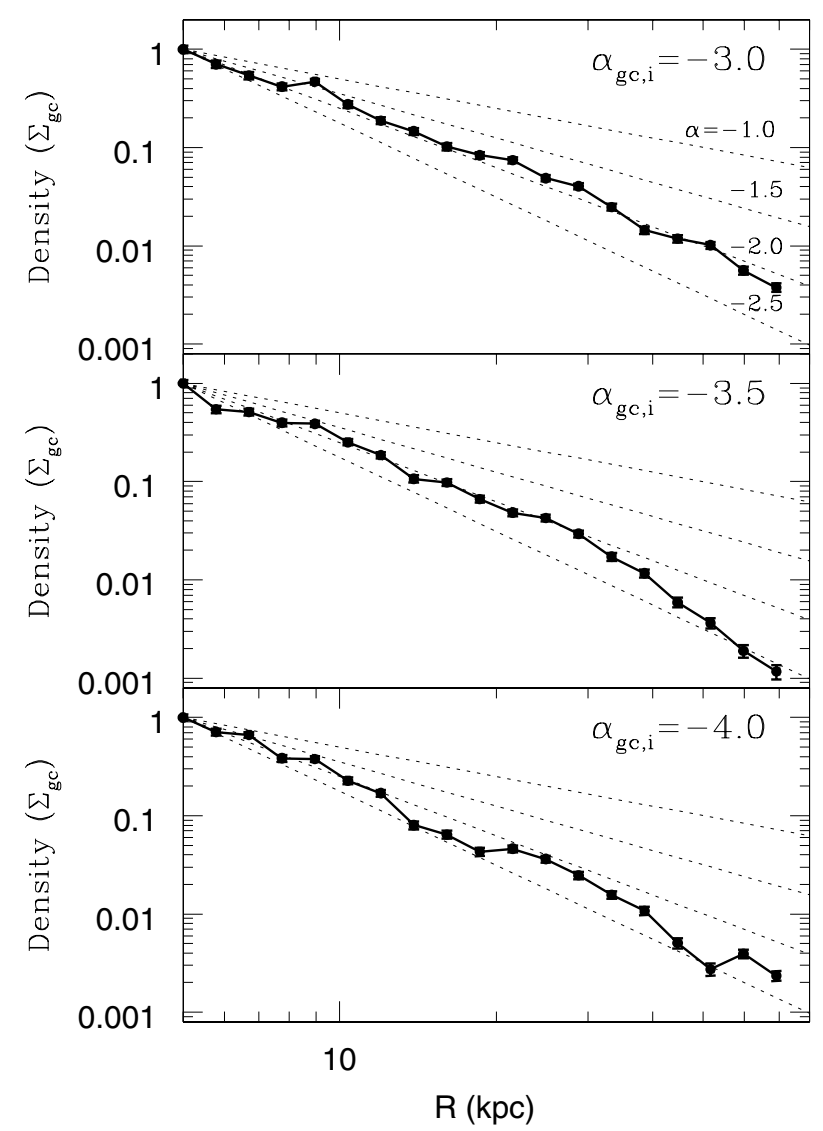

Fig. 5. Dependences of projected number distributions of GCs in merger remnants (i.e., elliptical galaxies) on the initial slope of radial density profiles of GCSs in spirals (i.e., $\alpha_{\mathrm{gc,i}}$ ) for the models with $N_{\mathrm{m}}=1$. For clarity, the density distributions are normalized to their central values. Thin dotted lines represent power-law slopes $(\alpha)$ of $\alpha=-2.5,-2.0,-1.5$, and -1.0 . Note that there are no remarkable differences between the three models.

Fig. 4. The derived luminosity-dependence of $r_{\mathrm{c}}$ is therefore steeper than the observed one.

\subsection{Robustness of the results}

\subsubsection{Dependence on $\alpha_{\mathrm{gc}, \mathrm{i}}$}

Although we adopt the slope of the Galactic GCS as the initial radial profile for GCSs in our progenitor spirals (i.e., $\alpha_{\mathrm{gc}, \mathrm{i}}=$ $-3.5)$, our results do not depend strongly on the initial profile slope. Figure 5 shows how the radial profiles of GCSs depend on $\alpha_{\mathrm{gc}, \mathrm{i}}$ for the models with $N_{\mathrm{m}}=1$. It is clear from this figure that there is no remarkable difference in the profiles between the three models for $R<20 \mathrm{kpc}$ : the difference of the final $\alpha_{\mathrm{gc}}$ between the models with $\alpha_{\mathrm{gc}, \mathrm{i}}=-3.0$ and -4.0 becomes at most $\sim 0.5$, if we fit the profiles to the power-law ones for $R<20 \mathrm{kpc}$. It should be also stressed that the model with $\alpha_{\mathrm{gc}, \mathrm{i}}=-3.5$ and -4.0 shows a slightly steeper profile for the outer parts of the merger remnants $(R>40 \mathrm{kpc})$ compared with the model with $\alpha_{\mathrm{gc}, \mathrm{i}}=-3.0$. This tendency can be seen in other models with different orbital configurations.

The derived weak dependence on $\alpha_{\mathrm{gc}, \mathrm{i}}$ is due to the fact that violent relaxation during major galaxy merging can effectively 




Fig. 6. The projected number distributions of stars (thin) and GCs (thick) in merger remnant (i.e., elliptical galaxies) with $N_{\mathrm{m}}=5$. For clarity, the density distributions are normalized to their central values. Thin dotted lines represent power-law slopes $(\alpha)$ of $\alpha=-2.5,-2.0$, -1.5 , and -1.0 .

wipe out the original density distributions of GCSs for $-4.0 \leq$ $\alpha_{\mathrm{gc}, \mathrm{i}} \leq-3.0$. The resulting profiles of $\alpha_{\mathrm{gc}} \sim-2$ of GCSs in merger remnants with $N_{\mathrm{m}}=1$ follow their dark matter halos, and furthermore do not depend on initial orbital configurations of galaxy merging. These results, combined with those shown in Fig. 1, imply that the present results on the dependence of $\alpha_{\mathrm{gc}}$ on $N_{\mathrm{m}}$ (thus on $M_{\mathrm{V}}$ ) does not depend strongly on $\alpha_{\mathrm{gc}, \mathrm{i}}$ and thus can be regarded as fairly robust.

\subsection{2. $N_{\mathrm{m}}>4$}

It is of interest to investigate whether the derived dependence of $\alpha_{\mathrm{gc}, \mathrm{i}}$ on $N_{\mathrm{m}}$ can be seen in models with $N_{\max }>4$ and thereby confirm that results are true for a possible range of $N_{\mathrm{m}}$. We note that the most massive ellipticals, with $M_{\mathrm{V}}<-23$ mag, are typically $\mathrm{cD}$ galaxies at the centres of clusters, for which accretion of GCs from cluster member galaxies are important (e.g., Bekki et al. 2003) and may well play a role in determining the radial profiles of GCSs. The luminosity range (for $N_{\max } \leq 4$ ) in the present simulations is thus appropriate for most ellipticals but not cD galaxies.

Figure 6 shows the radial density profiles of stars and GCs in the model with $N_{\mathrm{m}}=5$ for the PR orbital configuration. It is clear from this figure and Fig. 1 that the profile of GCS becomes even flatter than that in the model with $N_{\mathrm{m}} \leq 4$. This result can be seen in other models with different orbital configurations. For example, $\alpha_{\mathrm{gc}}$ is estimated as -0.77 for the PR and -1.03 for the PP models. These results confirm that radial density profiles of GCSs of ellipticals formed by sequential major merging depend strongly on $N_{\mathrm{m}}$. We note that for $N_{\max } \geq 5$ and $M_{\mathrm{d}}=4.0 \times 10^{10} M_{\odot}$, the resulting merger remnant has $M_{\mathrm{V}}<-24$ mag and thus few if any observational counterparts.

\subsubsection{Spatial coverage of GCSs}

It should be noted that the spatial coverage and number of GCs observed for the estimation of $\alpha_{\mathrm{gc}}$ (e.g., Ashman \& Zepf 1998) varies from galaxy to galaxy. The spatial coverage of the simulation data, on the other hand, is fixed at $5 \leq R \leq 20 \mathrm{kpc}$ for the $\alpha_{\mathrm{gc}}$ estimation, which suggests that the present compassion is not fully self-consistent. It is accordingly important for the present study to show how the $\alpha_{\mathrm{gc}}$ values depend on the spatial coverage. It is found that $\alpha_{\mathrm{gc}}$ is $\approx-2.0$ for $5 \leq R \leq 20 \mathrm{kpc}$, $\approx-1.6$ for $5 \leq R \leq 10 \mathrm{kpc}$, and $\approx-2.4$ for $10 \leq R \leq 20 \mathrm{kpc}$ in the HI model with $N_{\mathrm{m}}=1$. This result suggests that (1) $\alpha_{\mathrm{gc}}$ can be larger (i.e., GCS density profiles are flatter) if $\alpha_{\mathrm{gc}}$ is derived for the inner regions of ellipticals and (2) $\alpha_{\mathrm{gc}}$ can be smaller (i.e., GCS density profiles are steeper) if $\alpha_{\mathrm{gc}}$ is derived for the outer parts of ellipticals. Given the fact that these tendencies can be seen in other models (PP, RR, and LA) the present results imply that a more careful comparison between observations and simulations is necessary in deriving physical meaning of the GCS density profiles in ellipticals.

\section{Discussion}

In the present study we suggest that sequential major merging has the physical effect of flattening the radial density profiles of GCSs so that the radial profiles of GCSs in ellipticals contain fossil information about their past merger history. Neither of the above two points has been previously suggested by theoretical studies.

The GCSs in ellipticals are thought to be subject to dynamical destruction processes (e.g. Baumgardt 1998; Fall \& Zhang 2001; Vesperini et al. 2003), which are not included in this work. However there is currently little convincing observational evidence for destruction of GCs in ellipticals, e.g. Harris et al. (1998) found no variation of the bright end of the GCLF with galactocentric radius in M 87. Vesperini et al. (2003) also found no variation in the GCLF with radius in M 87, which they claimed was consistent with dynamical destruction if the GC mass function initially had a bell-shaped distribution.

To date, theoretical studies which focus on GC dynamical destruction have not yet investigated the luminosity dependence of the GCS density slope $\left(\alpha_{\mathrm{gc}}\right)$. Thus it remains to be seen whether GC destruction can account for this dependence, which is modelled here by sequential merging.

Destruction processes are expected to be stronger in the galaxy inner regions and thus play some role in determining the inner core radius of the GCS. However it is difficult to understand how such processes could explain the trend of $r_{\mathrm{c}}$ with galaxy luminosity given that destruction processes are expected to be less efficient in the central regions of more massive ellipticals (Murali \& Weinberg 1997).

Although the present sequential merger model can qualitatively explain some of the observed GCS structural properties, it has the following three disadvantages: firstly, it remains unclear why GCSs in some intermediate luminosity ellipticals $\left(M_{\mathrm{v}}<-21.5\right)$ can show steeper $\left(\alpha_{\mathrm{gc}}<-2.0\right)$ profiles, which are not fully consistent with the observation (See Fig. 2). Secondly, the simulated core radius $\left(r_{\mathrm{c}}\right)$ of a GCS for a given luminosity is appreciably larger than the observed one (Note that the derived luminosity-dependence of $r_{\mathrm{c}}$ is steeper than the observed one in Fig. 4). Thirdly, although the derived trend that $\alpha_{\mathrm{gc}}$ is always larger than $\alpha_{\mathrm{s}}$ is broadly consistent with the observed trend (e.g., Harris 1991), GCSs in some ellipticals show $\alpha_{\mathrm{gc}} \approx \alpha_{\mathrm{s}}$ (e.g., Ashman \& Zepf 1999), which is not fully consistent with the present results. 
What additional physics is required to overcome these limitations? As mentioned above, the inclusion of destruction processes may be important but this is unlikely to explain the trends with host galaxy luminosity. The present study is dissipationless and so does not include the formation of the new GCs, which would be formed preferentially in the central regions of gaseous galaxy mergers along with field star formation (e.g. Bekki et al. 2002). A future study should investigate the role of new GC formation in shaping the radial density profiles of GCSs. Given our lack of understanding of the physics of $\mathrm{GC}$ formation, this is a difficult task. As we have noted above, the contribution of new GCs to the existing GCSs of ellipticals appears to be small.

Bekki et al. (2003) showed that the strong tidal field in galaxy clusters can strip GCs from cluster ellipticals and consequently steepen the radial density profiles of the remaining GCS in the donor galaxy. Consequently, the recipient galaxy may obtain a flatter GCS profile.

Recent theoretical and numerical studies suggest that if cosmic reionization can strongly suppress the formation of old, metal-poor GCs in dwarfs at high redshifts $(z>6)$, then the radial profiles of GCSs in galaxies would be influenced at very early epochs (Santos 2003; Bekki 2005).

Finally, the accretion of GCs via minor mergers (discussed in the next section) may also have an important effect on some galaxies.

Thus the radial density profiles of GCSs in galaxies could be influenced by several physical processes associated with galaxy formation and evolution. Future studies should therefore seek to determine the relative importance of each physical mechanism in shaping the radial density profiles of GCSs. For this purpose, it is vital that future observational studies provide observational data on structural, kinematical, and chemical properties of GCs which can be compared with any theoretical predictions. For example, systematic observations of the radial dependence of GC luminosity functions in ellipticals over a range of galaxy luminosities will provide a clue as to the dominant physical processes.

\subsection{Minor mergers}

Although minor galaxy merging between a spiral and a dwarf can not form an elliptical (e.g., Walker et al. 1996; Bekki 1998), the accretion of GCs from the dwarf onto the spiral (e.g. Forbes et al. 2004) can possibly change the original density profiles of the GCS of the spiral. Since full discussions on this matter are obviously beyond the scope of this paper, we here briefly discuss how GC accretion events can change the radial profiles of GCSs of galaxies in a more general way. Although the following discussions are on GC accretion onto disk galaxies, the basic results can be also applied to GC accretion onto elliptical galaxies (Bekki \& Forbes 2005). Here we model a dwarf elliptical (dE) composed of dark matter, stars, and GCs and investigate the dynamical evolution of GCs during its orbital evolution around the present disk galaxy model. The full details of the $\mathrm{dE}$ model orbiting a spiral like the Galaxy or M31 can be

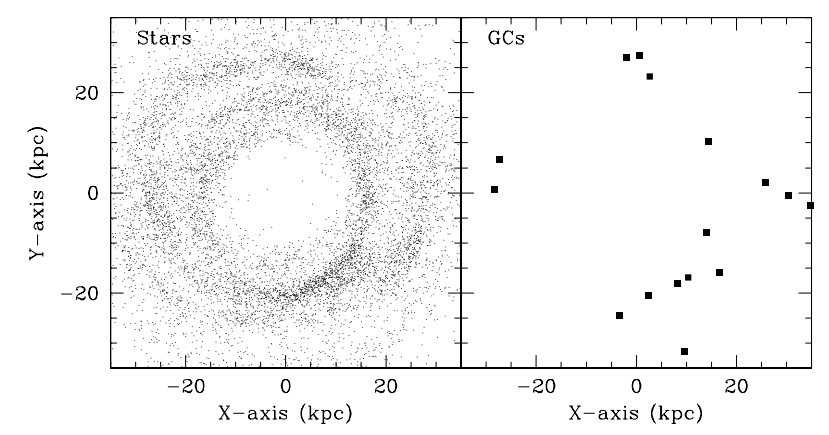

Fig. 7. Final distributions of stars (left) and GCs (right) projected onto the $x-y$ plane for the disk galaxy model after minor merging of the disk with a dwarf elliptical galaxy (dE) with $M_{\mathrm{B}}=-16 \mathrm{mag}$. GCs in the right panel for this minor merger model are those stripped from the $\mathrm{dE}$ and represented by filled squares. The center of the disk galaxy with the initial size of $17.5 \mathrm{kpc}$, which is not shown within the two frames for clarity, is coincident with the center of each frame. Note that most GCs are located in the outer part of the disk.

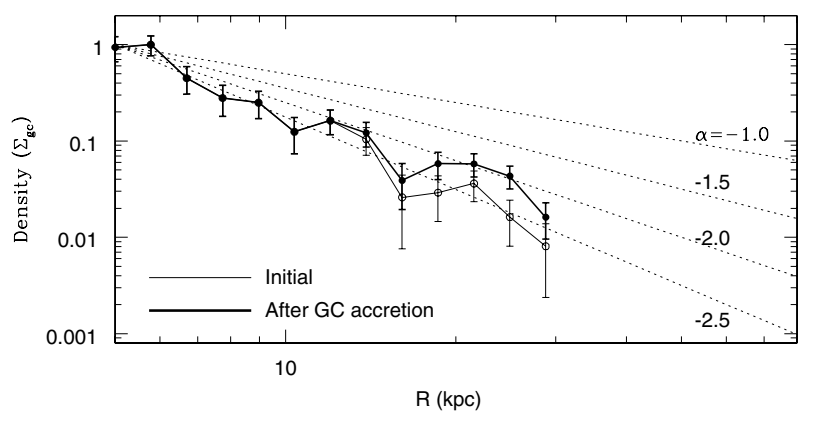

Fig. 8. The projected number distributions and GCs in the initial disk model (left) and the minor merger one (right) where a dwarf elliptical galaxy (dE) merges with the disk. For clarity, the density distributions are normalized to their central values. Thin dotted lines represent power-law slopes $(\alpha)$ of $\alpha=-2.5,-2.0,-1.5$, and -1.0 . Note that the outer profile $(R \sim 20 \mathrm{kpc})$ of the GCS of the disk becomes flattened after $\mathrm{GC}$ accretion associated with the minor merging.

found in Bekki \& Freeman (2003), Bekki \& Chiba (2004), and Bekki \& Forbes (2005).

Figure 7 shows the final distributions of stars and GCs initially within a dE with $R_{\mathrm{e}}=0.8 \mathrm{kpc}, M_{\mathrm{B}}=-16 \mathrm{mag}$, and $S_{\mathrm{N}}=5$ (corresponding to $20 \mathrm{GCs}$ in the $\mathrm{dE}$ ), where $S_{\mathrm{N}}$ is the specific frequency of GCs. In this model, the $\mathrm{dE}$ is assumed to be initially located $35 \mathrm{kpc}$ from the center of the disk galaxy and have a circular velocity of $209 \mathrm{~km} \mathrm{~s}^{-1}$ at this position. The $\mathrm{dE}$ can be almost completely destroyed within $\sim 6 \mathrm{Gyr}$ of its evolution around the disk galaxy, and GCs initially within the $\mathrm{dE}$ are all tidally stripped and dispersed into the halo region of the disk galaxy. The tidal destruction of the $\mathrm{dE}$ can happen well outside the disk in this model so that most GCs of the dE can be distributed in the outer part of the disk's halo. Consequently, the radial density profile of the GCSs composed both of the original GCs of the disk and of the stripped GCs from the dE in the disk can become flattened, as shown in Fig. 8.

These results imply that GC accretion associated with destruction of dwarfs in disk galaxies can flatten the radial profiles of GCSs in the disk galaxies. These results furthermore suggest that although minor merging can not transform disk 
galaxies into ellipticals (Walker et al. 1996; Bekki 1998), the radial profiles of GCSs in disk galaxies can be significantly changed by minor merger events if such events are repeated in the dynamical histories of the galaxies. The density profiles of dark matter halos of galaxies can be the key parameters in the flattening of the density profiles of GCSs by GC accretion (Bekki \& Forbes 2005, in preparation). Therefore the flattening processes by GC accretion are unlikely to depend on morphological types (e.g., Sp, E, and $\mathrm{cD}$ ) of luminous components, if all galaxies have similar dark matter profiles like the NFW profile. Recent numerical simulations have suggested that GC accretion from cluster member galaxies onto the central giant cDs in clusters of galaxies is highly likely (e.g., Bekki et al. 2003). The results shown in Figs. 7 and 8 therefore can suggest that GC accretion can be also responsible for the origin of the very flat density profiles of GCSs in cDs.

\section{Conclusions}

We have demonstrated that the observed luminosity dependence of the GCS slope $\left(\alpha_{\mathrm{gc}}\right)$ can be reasonably well reproduced in elliptical galaxies formed through sequential dissipationless major merger events. Previous numerical simulations have already demonstrated that the origin of structural nonhomology in elliptical galaxies can be closely associated with the dynamics of major galaxy merging (e.g., Capelato et al. 1995; Bekki 1998; Dantas et al. 2002). We suggest that structural non-homology seen in both the stellar component and GCSs of ellipticals can have a common origin, i.e. the growth of elliptical galaxies through dissipationless major merging. The derived $M_{\mathrm{V}}-r_{\mathrm{c}}$ and $\alpha_{\mathrm{gc}}-\alpha_{\mathrm{s}}$ relationships are also the direct result of this growth. Thus the radial density profiles of GCSs in elliptical galaxies can be regarded as containing fossil records of their merging histories. Given the fact that dynamical non-homology can be closely associated with the origin of the Fundamental Plane (e.g., Djorgovski \& Davis 1987) of elliptical galaxies, it is an interesting observational question whether GCSs show an analogous "Fundamental Plane".

Acknowledgements. We are grateful to the referee for valuable comments, which contribute to improve the present paper. K.B. and DAF acknowledge financial support from the Australian Research Council (ARC) throughout the course of this work. The numerical simulations reported here were carried out on GRAPE systems kindly made available by the Astronomical Data Analysis Center (ADAC) at National Astronomical Observatory of Japan (NAOJ).

\section{References}

Ashman, K. M., \& Zepf, S. E. 1992, ApJ, 384, 50

Ashman, K. M., \& Zepf, S. E. 1998, in Globular cluster systems (Cambridge, U. K.; New York: Cambridge University Press)

Battistini, P., Bonoli, F., Casavecchia, M., et al. 1993, A\&A, 272, 77 Baumgardt, H. 1988, A\&A, 330, 480

Beasley, M. A., Baugh, C. M., Forbes, D. A., Sharples, R. M., \& Frenk, C. S. 2002, MNRAS, 333, 383
Beasley, M. A., Forbes, D. A., Brodie, J. P., \& Kissler-Patig, M. 2004, MNRAS, 347, 1150

Bekki, K. 1998, ApJ, 496, 713

Bekki, K. 2005, ApJ, 626, L93

Bekki, K., \& Freeman, K. C. 2003, MNRAS, 346, L11

Bekki, K., \& Chiba, M. 2004, A\&A, 417, 437

Bekki, K., \& Forbes, D. A. 2005, in preparation

Bekki, K., Forbes, D. A., Beasley, M. A., \& Couch, W. J. 2002, MNRAS, 335, 1176

Bekki, K., Forbes, D. A., Beasley, M. A., \& Couch, W. J. 2003, MNRAS, 344, 1334

Bekki, K., Beasley, M. A., Brodie, J. P., \& Forbes, D. A. 2005, MNRAS, submitted

Bell, E., et al. 2005, [arXiv: astro-ph/0506425]

Capelato, H. V., de Carvalho, R. R., \& Carlberg, R. G. 1995, ApJ, 451, 525

Cohen, J. G., Blakeslee, J. P., \& Ryzhov, A. 1998, ApJ, 496, 808

Cohen, J. G., Blakeslee, J. P., \& Côte, P. 2003, ApJ, 592, 866

Dantas, C. C., Capelato, H. V., Ribeiro, A. L. B., \& de Carvalho, R. R. 2003, MNRAS, 340, 398

Djorgovski, S., \& Davis, M. 1987, ApJ, 313, 59

Djorgovski, S., \& Meylan, G. 1994, AJ, 108, 1292

Fall, S. M., \& Zhang, Q. 2001, ApJ, 561, 751

Forbes, D. A., Franx, M., Illingworth, G. D., \& Carollo, C. M. 1996, ApJ, 467, 126

Forbes, D. A., Brodie, J. P., \& Grillmair, C. J. 1997, AJ, 113, 1652

Forbes, D. A., Beasley, M. A., Brodie, J. P., \& Kissler-Patig, M. 2001, ApJ, 563, L143

Forbes, D. A., Strader, J., \& Brodie, J. P. 2004, AJ, 127, 3394

Freeman, K. C. 1993, in the globular cluster-galaxy connection ed. G. H. Smith, \& J. P. Brodie (San Francisco: ASP), ASP Conf. Ser., 48,27

Grillmair, C. J., Faber, S. M., Lauer, Tod R., et al. 1994, AJ, 108, 102

Harris, W. E. 1976, AJ, 81, 1095

Harris, W. E. 1986, AJ, 91, 822

Harris, W. E. 1991, ARA\&A, 29, 543

Harris, W. E., \& Racine, R. 1979, ARA\&A, 17, 241

Harris, W. E., Harris, G., \& McLaughlin, D. 1998, AJ, 115, 1801

Kuntschner, H., Ziegler, B. L., Sharples, R. M., Worthey, G., \& Fricke, K. J. 2002, A\&A, 395, 761

Murali, C., \& Weinberg, M. D. MNRAS, 288, 767

Navarro, J. F., Frenk, C. S., \& White, S. D. M. 1996, ApJ, 462, 563

Pierce, M., Brodie, J. P., Forbes, D. A., Beasley, M. A., \& Strader, J. 2005, MNRAS, accepted

Santos, M. R. 2003, Extragalactic Globular Cluster Systems ed. M. Kissler-Patig (Garching: ESO), 348

Strader, J., Brodie, J. P., \& Forbes, D. A. 2004, AJ, 127, 295

Strom, S. E., Strom, K. M., Wells, D. C., et al. 1981, ApJ, 245, 416

Sugimoto, D., Chikada, Y., Makino, J., et al. 1990, Nature, 345, 33

Toomre, A. 1977, in The evolution of galaxies and stellar Populations ed. by B. Tinsley, \& R. Larson (New. Haven. CN: Yale Univ. Press), 401

van den Bergh, S. 2000, The Galaxies of the Local Group (Cambridge: Cambridge Univ. Press)

Vesperini, E., Zepf, S. E., Kundu, A., \& Ashman, K. M. 2003, ApJ, 593, 760

Walker, I. R., Mihos, J. C., \& Hernquist, L. 1996, ApJ, 460, 121

Whitmore, B. C., \& Schweizer, F. 1995, AJ, 109, 960

Zepf, S. E., \& Ashman, K. M. 1993, MNRAS, 264, 611

Zinn, R. 1985, ApJ, 293, 424 